\title{
La grammatica lulliana dal trivium all'Arte
}

\author{
Elena Pistolesi
}

Università di Modena e Reggio Emilia

elena.pistolesi@unimore.it

\begin{abstract}
La grammatica ha un ruolo marginale nel progetto di rifondazione dei saperi che impegna Llull fin dal 1290. Mentre retorica e logica sono oggetto di due opere specifiche (Rhetorica nova del 1301 e Logica nova del 1303), la grammatica viene sondata attraverso i principi artistici solo nell'Ars generali ultima (1305-8) all'interno delle 'Cento forme'. L'articolo ripercorre le tappe di questa inclusione attraverso le definizioni della grammatica offerte dal corpus lulliano, discute l'ipotesi di un orientamento speculativo della disciplina ed evidenzia l'importanza del declinare come fondamento dell'analogia tra i modi della grammatica e i principi correlativi che strutturano l'ontologia dinamica dell'Arte. I riscontri testuali mostrano che la grammatica rappresenta un altro capitolo della «dispersa» filosofia del linguaggio di Llull, lontana nei fini e nell'articolazione dalla prospettiva dei Modisti.

Parole chiave: Llull; grammatica; Ars; grammatica speculativa; filosofia del linguaggio.
\end{abstract}

\begin{abstract}
The grammar plays a marginal role in the process of re-foundation of knowledge that Ramon Llull engages since 1290 . While rhetoric and logic are the subject of two specific works (Rhetorica nova of 1301 and Logica nova of 1303), the grammar is probed through the artistic principles only in Ars Generalis Ultima (1305-8) within the 'Hundred forms'. The paper traces the stages of this inclusion through the definitions given in the Lullian corpus, discusses the hypothesis of a speculative orientation of the discipline and underlines the importance of declinare as the basis of analogy between the grammar's modi and correlative principles that structure the dynamic ontology of Art. The findings show that grammar is another chapter of the "dispersed» Lullian philosophy of language, far from the articulation and purposes of the Modistic model.
\end{abstract}

Keywords: Llull; Grammar; Ars; Speculative Grammar; Philosophy of Language. 
Sulla grammatica nell'opera di Ramon Llull esiste una bibliografia circoscritta, che riguarda principalmente due aspetti: da un lato le diverse accezioni della parola, ${ }^{1}$ dall'altro la sua interpretazione in chiave filosofica. ${ }^{2}$ Si passa pertanto dal vocabolario relativo alla grammatica, intesa come sinonimo di latino e delle sue regole o come primo gradino di accesso al sapere, alla definizione di una semiotica specifica, che eccede i limiti della disciplina per proporsi come possibile chiave di lettura dell'intero sistema lulliano. Rispetto a questi due punti di vista, la nostra ricognizione seguirà le tappe dell'inclusione della grammatica nel sistema artistico, attraverso il filo interno delle citazioni e il confronto con la terminologia coeva, per verificare le ipotesi di un suo orientamento speculativo.

I testi commentati, riportati in ordine cronologico nella Tavola posta in appendice, derivano dalla consultazione del Glossari general lul.lià di Miquel Colom (Palma de Mallorca: Ed. Moll, 1982-1985) e del CETEDOC Library of Christian Latin Texts (CLCLT)-6, nel quale si possono interrogare le opere latine edite nel ROL. ${ }^{3}$ La prima definizione in ordine di tempo è contenuta nella Doctrina pueril (1274-6):

[1] Gramatica es dretament parlar e escriure, e per assó es aleta a esser comú lenguatge a les gents qui per luyetat de terres e de participació son desvariables en lur lenguatge.

[2] Fil, si vols apendre gramatica .iii. cozes te convenen a saber: costrucció, declinació e vocables [...]

[3] Cor aurás apresa gramatica en est libre, enaprés aprin-la en lo Libre de defenicions e de questions per so que anans ages les altres sciencies. Si en nula art ne sciencia vols entrar, primerament te cové a passar per esta art de gramatica, qui es portal per lo qual hom passa a saber les altres sciencies. ${ }^{4}$

Il passo, dopo una definizione tradizionale della disciplina (dretament parlar e escriure), riproposta letteralmente nell'Ars generalis ultima e nell'Ars brevis,

1. Miquel Colom Mateu, «Aspectes secundaris dins l'obra escrita de Ramon Llull ("Sabaters i Sabates", "Teles i Vestits", "Gramàtica”)», Estudios Lulianos, n. 17, 1973, p. 43-60; Lola BADIA, «A propòsit de Ramon Llull i la gramàtica», in ID., Teoria i pràctica de la literatura en Ramon Llull, Barcelona: Quaderns crema, 1992, p. 173-194. Il lavoro di Lola Badia individua tre usi del termine, in parte sovrapposti: grammatica intesa come sinonimo di 'latino', come disciplina che contiene le norme per l'insegnamento e per l'apprendimento del latino e, infine, arte cui si applicano i principi generali svolti nell'Ars generalis ultima (1305-8).

2. Questa prospettiva è svolta in Ramon Llull, Retòrica nova, a cura di Josep Batalla, Lluís Cabré i Marcel Ortín; pres. Anthony Bonner; pr. Robert D.F. Pring-Mill, Turnhout: Brepols - Santa Coloma de Queralt: Edèndum, 2006 (= TORL 1), a p. 52 e in alcune note del commento. Tali riscontri, di natura prevalentemente lessicale, sono alla base dell'interpretazione di Mauricio Bешснот, «Some Features of the Semantics in the Lullian Tradition", in Alexander Fidora e Carles Sierra (a cura di), Ramon Llull: From the Ars Magna to Artficial Intelligence, Barcelona: IIIA-CSIC, 2011, p. 107-116, che sarà discussa alla fine di questo lavoro.

3. Lo schema si limita alle opere che contengono una definizione della grammatica entro il novero delle arti, fra le quali consideriamo anche l'elenco delle 'Cento forme'.

4. NEORL VII, 2005, p. 189-190. Mio il corsivo, qui come nelle successive citazioni, salvo diversa indicazione. 
fa riferimento alla lingua latina (aleta significa «eletta», «scelta» come linguaggio comune), alla norma (costrucció, declinació e vocables) e al suo carattere propedeutico (portal). ${ }^{5} \mathrm{Il}$ contesto è quello delle arti liberali, alle quali l'autore introduce il figlio, secondo un topos consolidato della letteratura didattica.

L'individuazione del latino come lingua di comunicazione tra genti lontane (comú lenguatge) è svolta anche nel noto capitolo del Blaquerna (1276-83), «Tu solus, Dominus», nel quale alla diversità di linguaggi (desvariació de lenguatges) si imputano il fallimento della predicazione, l'origine delle guerre e la formazione di sette. Alla domanda su quale sia il metodo da seguire per eliminare tale varietà e «a qual lenguatge sseria millor que hom feés convenir totes les gents en general» perché possano comprendersi, l'apostolo risponde che, affinché nel mondo ci siano una sola lingua e un solo credo, lo strumento migliore è l'adozione del latino, "cor latí es lo pus general lenguatge e en latí ha moltes paraules d'altres lenguatges e en latí son nostres libres» (NEORL VIII, 2009, p. 414-415). Con una sola lingua gli uomini impareranno a comprendersi e, «per l'enteniment, amar-s'an e pendran-ne mills semblans custumes, en les quals se concordaran» (ibid.p. 416). ${ }^{6}$ L'utopia della soluzione proposta nel Blaquerna, che accompagna le più convinte iniziative lulliane sulla fondazione di scuole dove $\mathrm{i}$ missionari potessero apprendere le lingue degli infedeli, è sottolineata dal contestuale richiamo all'unità linguistica originaria: l'apostolo chiede infatti ai cardinali che lo aiutino a trattare "con tots los lenguatges qui son puscam tornar a .i. tant solament». Insieme a un breve accenno all'episodio babelico contenuto nella Doctrina pueril, ${ }^{7}$ questo è il solo riferimento alla diversificazione delle lingue reperibile nella produzione lulliana. ${ }^{8}$

5. La propedeuticità della grammatica è confermata anche nel Blaquerna (1276-83), quando si racconta la formazione del protagonista («Blaquerna aprengué tanta gramàtica que sabia entendre i parlar llatí», NEORL VIII, 2009, p. 95). La grammatica come insieme di regole non pare essere esclusiva del latino, se Llull parla nell'Arbre de ciència di «gramatica dels latins» (ORL XIII, 1926, p. 250) e nel Liber de passagio di "grammaticam nostram» (ROL XXVIII, 2003, p. 343).

6. Un concetto simile è espresso nel prologo della Rhetorica nova in merito alla comunicazione ornata: "Quanto vero sunt placibiliora [verba], tanto necesse est ut per ipsa loquentes et audientes in finem concorditer uniantun, ROL XXX, 2005, p. 22. Sulla grammatica come mezzo per conseguire l'armonia, cfr. Marta M.M. Romano e Óscar De la Cruz, "The Human Realm», in Alexander Fidora, Josep. E. Rubio (a cura di), Raimundus Lullus. An Introduction to his Life, Works and Thought, Turnhout: Brepols 2008 (ROL, Supplementum Lullianum, II), p. 363-459, a p. 405.

7. «Mas, ans que la torra fos ten alta com els la volien fer, tramés Deus a aquels qui la torra faien diverses lenguatges, enaxí que los uns no entenien los altres; e per assó no pogren puyar la torra segons que volgren; e ladoncs comensá la diversitat dels lenguatges que ara son» (NEORL VII, 2005, p. 271). Un accenno indiretto all'episodio di Babele è segnalato da Lola BADIA, «Monolingüisme i plurilingüisme segons Ramon Llull: de l'ideal unitari a les solucions pragmàtiques», Boletín de la Real Academia de Buenas Letras de Barcelona, n. 43, 1991-1992 (1993), p. 277-295, a p. 279.

8. Sul passo del Blaquerna scrive Joan Martí i CASTell, «Ramon Llull, creador de la llengua literària», $S L$, n. 35, 1995, p. 31-49, a p. 43: «És cert, nogensmenys, que reconèixer el caràcter d'universalitat de la llengua llatina és en realitat la manifestació d'una actitud 
Nell'Arbre de ciència (1295-6) la definizione di grammatica non si discosta dagli esempi visti fin qui, anche se essa viene considerata all'interno delle altre arti liberali e meccaniche quale espressione dell' habitus artificialis, cioè delle attività extra naturam. Oltre all'identificazione con il latino e la sua norma, le questions contenute nella stessa opera specificano che la grammatica costituisce la base della retorica e che è meno generale della logica perché necessita di più parole. ${ }^{9}$

\section{Arte e scienza}

La seconda occorrenza in ordine cronologico, tratta dalla Taula general (12934), ci immette in un ambito diverso, che riguarda la relazione dell'Arte lulliana come scientia generalis (attributo che compare qui per la prima volta) ${ }^{10} \mathrm{con}$ gli altri saperi, che insistono su principi particolari. A partire dalla Taula vediamo alternarsi i termini arte e scienza, senza che a tale oscillazione si possa attribuire un significato specifico, dal momento che investe più discipline $\mathrm{e}$ che resta fluida nel tempo. ${ }^{11}$ Il riferimento alla grammatica come 'scienza' ritorna infatti nella Lectura brevis (1304), nel Liber de refugio intellectus (1308) e nell'Ars mystica (1309), mentre nell'Ars generalis ultima (d'ora in poi indicata con la sigla $A G U$ ) e nell'Ars brevis essa viene detta ars. La Lectura Artis quae intitulatur Brevis practica Tabulae generalis fa parte di un gruppo di scritti composti tra Genova e Montpellier, negli anni 1303 e 1305, nel quale rientra anche la prima parte dell' $A G U$, completata a Pisa nel $1308 .{ }^{12}$ Dall'applicazione svolta nella Lectura («Primo ostenditur quo modo artista sciat applicare principia et regulas huisus Artis, quae generalia aut universalia sunt, ad prin-

diglòssica no superada del tot. Però diglòssia real —almenys latent— a part, és evident que l'intent de comptar amb una única llengua per a la unificació de la humanitat amb el cristianisme és una prova més de la identitat llengua/pensament».

9. «De les questions de gramàtiga. 174 Quest. Demanà lermità a Ramon si gramàtiga fo enans considerada per retòric que per gramàtic. Solució. Enaxí es rectòrica fi de gramàtiga com habitar de cambra. (...) 176 Quest. Ramon, per que lo gramàtic usa més de nom e de verb que lo lògic? Sol. Sciencia qui es pus general no ha necessitat de tants termens com altra sciencia» (ORL XIII, 1925, p. 250).

10. Antony Bonner, The Art and Logic of Ramon Llull. A User's Guide, Leiden-Boston: Brill, 2007, p. 6.

11. Nell' $A G U$ ricorre, accanto ad ars e scientia, anche la parola facultas. Llull tende di solito a chiamare 'arti' le discipline del trivio, preferendo la definizione di 'scienza' per la teologia, il diritto e la medicina. La stessa alternanza è già presente in Boezio: cfr. Giulio D'Onofrio, «La scala ricamata. La philosophiae divisio di Severino Boezio tra essere e conoscere», in ID. (a cura di), La divisione della filosofia e le sue ragioni: lettura di testi medievali, VI-XIII secolo. Atti del settimo Convegno della Società italiana per lo studio del pensiero medievale (S.I.S.P.M.), Assisi, 14-15 novembre 1997, Cava de' Tirreni: Avagliano, 2001, p. 11-63, a p. 19, n. 21.

12. Sui problemi di datazione dell'opera, cfr. Antoni Bonner, «Correccions i problemes cronològics», SL, n. 35, 1995, p. 85-95; Introducció a Ramon Llull, Disputa entre la fe i l'enteniment, a cura di Josep Batalla e Alexander Fidora (TORL, 3), Tournhout - Santa Coloma de Queralt: Brepols - Obrador Edèndum - Publicacions URV, 2011, p. 17. Sulle opere di questa fase, cfr. Bonner, The Art and Logic, cit., p. 8. 
cipia aliarum scientiarum, quae subalternata sunt principiis et regulis huius Artis») discende l'analisi delle parti della filosofia, che comporta anche un breve accenno all'oggetto della grammatica:

Diuiditur autem philosophia in tres partes: Prima pars est de trinio, in quo agitur de sermone; secunda de quadriuio, in quo agitur de ente mathematico; et in tertia parte agitur de ente naturali. Triuium autem constituunt tres scientiae de sermone, scilicet, grammatica, dialectica et rhetorica.

Grammatica siquidem considerat in sermone congruum et incongruum; dialectica uerum et falsum; rhetorica ornatum et inornatum. ${ }^{13}$

Nella Lectura compaiono per la prima volta nel corpus lulliano la parola 'trivio' e la locuzione scientiae de sermone, comprendente grammatica, dialettica e retorica. ${ }^{14}$ Altre particolarità sono la definizione stessa della disciplina, che si discosta dalle precedenti ("Grammatica siquidem considerat in sermone congruum et incongruum»), e il ricorso al tecnicismo subalternata, ${ }^{15}$ che traduce implicata della Taula. Nonostante queste innovazioni, Llull accantona l'argomento, di cui non mostra l'applicazione, limitandosi ad esporre i principi della logica e della retorica "causa brevitatis».

Lo statuto della grammatica come arte o scienza è un tema centrale nella trattatistica coeva. ${ }^{16} \mathrm{Nel}$ XIII secolo i logici affrontano le discipline del trivio nel complesso della scientiae sermocinales, cominciando a distinguere tra ars grammaticae, nell'accezione normativo-retorica indicata da Prisciano, e scientiae grammaticae, cui si attribuisce il significato di 'ricerca linguistica'. ${ }^{17}$ Tra i molti esempi disponibili, basterà ricordare che le Quaestiones

13. ROL XX, 1995, p. 397.

14. La parola trivium ricorre solo tre volte nella produzione lulliana: due nella Lectura e una nel Liber de venatione substantiae, accidentis et compositi (1308): «Philosophus tractat de triuio et quadriuio. Et suum subiectum est intelligere» (ROL XXII, 1998, p. 127). Nel corso della Lectura Llull usa 'logica' in luogo di 'dialettica'. Sulla sostituzione della dialettica con la logica, cfr. Olga Weijers, «L'appelation des disciplines dans les classifications des sciences au XII ${ }^{\mathrm{e}}$ et au XIII ${ }^{\mathrm{e}}$ siècles», Bulletin du Cange. Archivum Latinitatis Medii Aevii, n. 46-47, 1988 , p. 39-64, a p. 54-55.

15. Sulla derivazione tomistica di questa terminologia e sul significato che assume nella speculazione lulliana, cfr. Josep Maria Ruiz Simon, L’Art de Ramon Llull i la teoria escolàstica de la ciència, Barcelona: Quaderns Crema, 1999. Sul rapporto tra arti e filosofia, Antoni Bordoy, La filosofía de Ramon Llull, Mallorca: Objeto Perdido, 2011, p. 29-34.

16. Se per Prisciano la grammatica è un'arte che tratta dei testi di poeti e prosatori, e in tal senso riflette la concezione della disciplina propria dell'età classica, a partire da Isidoro di Siviglia (con il precedente di Marziano Cappella) compare la definizione di 'scienza' (Etymologiae I, 5, 1-2), che avrà largo seguito fino al XII secolo.

17. Gian Carlo Alessio, "Sul De ortu scientiarum di Robert Kilwardby», in Giulio D'OnoFRIO (a cura di), La divisione della filosofia, cit., p. 107-135, a p. 133-134 osserva: «La predicazione con "sermocinalis" ("ratione sermonis de quo est") del gruppo di discipline che costituivano l'antico trivio, senza precedenti nell'età antica ed altomedievale [...], vale a disambiguare il termine "logica", interpretato "per ethimologiam", appare in Ugo di San Vittore e si stabilizza, pur senza divenire esclusivo (sono concorrenti "rationalis", "eloquen- 
Alberti de modis significandi (1285) si aprono con la domanda «Utrum grammatica sit scientia». ${ }^{18} \mathrm{Vi}$ si discute lo statuto della disciplina attingendo ai testi coinvolti nel più ampio dibattito sul dominio della filosofia e sulla costituzione delle scienze in senso aristotelico. Per i modisti la grammatica, intesa come indagine sulle cause del linguaggio, è scienza speculativa e teoretica, capace di descrivere la struttura universale comune a tutte le lingue, e nettamente distinta dalla grammatica normativa, che si occupa solo della loro veste superficiale (le voces o le loro figurationes). ${ }^{19}$ Termini analoghi nella forma, ma distanti nella sostanza, si riscontrano nel Liber de refugio intellectus (1308). Per risolvere la contraddizione apparente tra premessa maggiore e minore in cui Llull ha formalizzato la nuova fallacia, ${ }^{20}$ alla domanda «Vtrum de sermone sit scientia?», la risposta risulta negativa se interpretiamo il sermone come vox, ma è positiva se riferita al contenuto, "cum quo intellectus facit scientiam de sermone». La domanda successiva, «Vtrum scientia de sermone sit necessaria?», è risolta nel modo seguente:

Solutio: Nulla scientia de sermone est necessaria. Sed grammatica est scientia de sermone; ergo grammatica non est necessaria.

Nulla scientia de sermone est necessaria. Sed quaedam scientia de sermone est necessaria; ergo quaedam scientia de sermone est necessaria et non est necessaria.

Maior est duplex: Non est necessaria, propter hoc quia est a contingentia. Verumtamen est necessaria causa bene uiuendi, scilicet recte loquendi. Minor est uera; quia ponendo etc. ${ }^{21}$

In questo caso, se intendiamo la scientia de sermone come priva di finalità (nella Taula general si legge: "Contingencia es aventura»), ${ }^{22}$ la risposta sarà negativa. Diviene positiva in rapporto alla intentio, identificata con il bene

tia" e persino "trivium"), nella dottrina a partire, sembra, dal quinto decennio del XIII secolo". Fra gli esempi viene citato anche il Liber de universalibus, qui attribuito a Ramon Llull, in realtà opera apocrifa. Per la documentazione terminologica, cfr. Helène MerLe, "Ars», Bulletin de philosophie médiévale, n. 28, 1986, p. 95-133, a p. 116-117. Sul passaggio dall'ars grammatica alla sermocinalis scientia, cfr. Martin Irvine e David Thomson, "Grammatica and literary theory», in Alastair Minnis e Ian Johnson (a cura di), The Cambridge History of Literary Criticism, vol. 2: The Middle Ages, Cambridge: Cambridge University Press, 2005, p. 24-30.

18. Per questa evoluzione, si veda l'introduzione di Louis G. Kelly a Pseudo-Albertus Magnus, Quaestiones Alberti de modis significandi: a critical edition, translation and commentary of the British Museum incunabulum C.21.C.52 and the Cambridge incunabulum Inc.5.J.3.7, Amsterdam: Benjamin, 1977.

19. Alfonso Maierù, «La grammatica speculativa», in C. Leonardi e G. OrLandi (a cura di), Aspetti della letteratura latina nel secolo XIII, Firenze: La nuova Italia, 1986, p. 147-167.

20. Cfr. Charles Lohr, Prolegomena al Liber de novis fallaciis (ROL XI, 1983, p. 3-6), e BonNER, The Art and Logic, cit., p. 233-243.

21. ROL XI, 1983, p. 260-261.

22. Anthony Bonner e Maria Isabel Ripoll, Diccionari de definicions lul.lianes / Dictionary of Lullian Definitions, Barcelona-Palma de Mallorca: Universitat de Barcelona-Universitat de les Illes Balears, 2002, s.v. contingencia (Col-lecció Blaquerna, 2). 
vivendi, ovvero con il recte loquendi. I termini correnti del dibattito, con la contrapposizione fra necessità e contingenza, vengono applicati qui a una dimensione diversa dalla ricerca delle cause che impegnava i filosofi del linguaggio contemporanei e sono ricondotti entro la dottrina lulliana delle due intenzioni che riguarda gli scopi dell'agire umano. ${ }^{23}$

Si è visto che nella Lectura compaiono per la prima il termine 'trivio' e la locuzione scientiae de sermone, collegata alla precisazione dei rapporti con le altre arti. Ancora nella Lectura si osserva una parziale divergenza rispetto alle precedenti definizioni per l'impiego delle nozioni di congruum et inconguum, anticipate parzialmente nella Taula. Secondo Prisciano (Institutiones II, 15) oratio è "ordinatio dictionum congrua», dove congrua significa "corretta» in contrapposizione a incongrua, sinonimo di «scorretta». Con "costruzione incongrua» i grammatici medievali intendono tanto quella che viola le regole grammaticali, quanto quella che va contro il senso logico. ${ }^{24} \mathrm{La}$ trattatistica distingue inoltre la costruzione semplicemente rispettosa della norma da quella retoricamente elaborata, che ricorre all'ordo artificialis delle parole, al cursus, ai tropi e al linguaggio metaforico. Il primo tipo è destinato alle persone comuni, il secondo ai sapienti. ${ }^{25}$ Nella divisione tra conguo e incongruo, che si faceva risalire a Donato e a Prisciano, ${ }^{26}$ la grammatica è precettiva quando considera il congruum, è proibitiva rispetto all'incongruum (identificato di solito con i vitia), e permissiva quanto alle figure. ${ }^{27} \mathrm{Il}$ termine congruitas è presente con un diverso significato, cioè con valore tecnico, nel lessico dei modisti. Tommaso di Erfurt lo usa con riferimento alla sintassi, che articola in tre livelli: la constructio, la congruitas e la perfectio. Oltre la combinazione delle parti del discorso, per ottenere la costruzione perfetta si dovrà infatti valutare la concordanza dei componenti di una frase, che riguarda tanto l'accordo dei modi significandi, quanto il contesto. ${ }^{28}$ Stando alle scarne informazioni presenti nella Lectura, la definizione lulliana si attiene all'accezione retorico-grammaticale ereditata dalla tradizione antica.

23. Sulla dottrina delle due intenzioni in Llull, cfr. Josp Maria Ruiz Simon, “"En l'arbre són les fuyles per ço que y sia lo fruyt": apunts sobre el rerafons textual i doctrinal de la distinció lul.liana entre la intenció primera i la intenció segona en els actes propter finem», $S L$, n. 42, 2002, p. 3-25. Sulla rivisitazione del bene esse in rapporto al linguaggio, cfr. Elena Pistolesi, "El rerefons de l'affatus lul.lià», in Joan Mas, Joan Miralles i Pere Rosselló (a cura di), Actes de l'Onzè Col.loqui Internacional de Llengua i Literatura Catalanes. Palma (Mallorca), 8-12 de setembre del 1998, vol. I, Barcelona: Publicacions de l'Abadia de Montserrat, 1998, p. 73-92.

24. Cfr. Charles Thurot, Extraits de divers manuscrits latins pour servir à l'histoire des doctrines grammaticales au Moyen Âge, Paris: Imprimerie impériale, 1869, p. 218-219 e 227-228.

25. Ibid., p. 459.

26. Ibid., p. 133.

27. Su questa tripartizione, cfr. L. BADIA, «A propòsit de Ramon Llull», cit., p. 191.

28. Sull'uso tecnico di congruitas si possono vedere Geoffrey L. Bursill-Hall, Speculative grammars of the Middle Ages. The doctrine of Partes orationis of the Modistae, The Hague - Paris: Mouton, 1971, p. 303-307; e C. Marmo, La semiotica del XIII: tra arti liberali e teologia, Milano: Bompiani, 2010, p. 58-66. 


\section{L'applicazione dell'Ars generalis ultima}

L'illustrazione completa della grammatica avviene nel contesto delle 'Cento forme', una lista ordinata di temi, che nell' $A G U$ ricevono un trattamento esteso basato sui tredici loci dell'Arte. ${ }^{29}$ Llull offre in tal modo un modello dettagliato di come si possa studiare ciascun concetto attraverso i Principi e le Regole del proprio sistema. ${ }^{30}$ Dopo la serie delle forme generalissime che vanno da 1 a 79 («entità», «essenza», «unità», "pluralità», ecc.), ha inizio la Applicatio Artis generalis ad Artes particulares, o subalternatae, che sono, nell'ordine, la teologia (definita scientia), la filosofia («subiectum in quo intellectus se contrahit ad omnes artes et scientias»), le artes (geometria, astonomia, aritmetica, musica, retorica, logica), inclusa la grammatica (forma 88), di cui si dà la seguente definizione:

De grammatica. Grammatica est ars ad inueniendum modum recte loquendi recteque scribendi.

Per definitionem differentiae grammatica diuiditur in nouem modos. Primus modus est de grammatica et de numero octo partium orationis. Secundus est de casu. Tertius de coniunctione. Quartus de declinatione. Quintus de genere. Sextus de regimine. Septimus de constructione. Octauus de orthographia. Nonus est de figura. ${ }^{31}$

Una volta distinta la grammatica dalle altre discipline sulla base dei nove modi suddetti, Llull espone le basi del suo svolgimento: «Grammatica est applicabilis siue applicanda huic Arti. Nam sicut grammatica docet modum recte loquendi recteque scribendi, sic ista Ars docet inuenire alias Artes». L'analogia costituisce il fondamento dell'intera dimostrazione e spiega le peculiarità dei nove modi rispetto alla disposizione della materia offerta dalla grammatica normativa: i casi diventano nell' $A G U$ voci indipendenti, invece di essere trattati entro le rispettive parti del discorso; la coningatio viene estratta dal verbo.

La successione delle parti del discorso non si discosta da quella tradizionale, condivisa anche dai grammatici speculativi, come si può osservare nello schema seguente:

29. Llull propone questa lista in più opere, modificandone l'assetto: Arbre de ciència (1295-6), Proverbis de Ramon (1296?), Logica nova (1303), AGU, Ars brevis (1308) e Ars consilii (1315). Sulle 'Cento forme' e sulla loro articolazione nell' $A G U$, cfr. il contributo di Lola BADIA in questo numero della rivista.

30. Cfr. Josep Enric Rubio, «Thought: the Art», in Alexander Fidora e Josep. E. Rubio (a cura di), Raimundus Lullus. An Introduction, cit., p. 243-310, a p. 293-294.

31. La trattazione completa della grammatica si trova in ROL XIV, 1986, p. 367-370, al quale si rinvia data la lunghezza del testo. Nell'indice si legge coniunctione, ma nel relativo capitolo si parla di coniugatione. L'apparato critico non registra varianti nella tradizione rispetto a questa discordanza.

32. Nell'esposizione l'ordine della preposizione e dell'avverbio è invertito. 


\begin{tabular}{|c|c|c|c|c|}
\hline $\begin{array}{l}\text { Llull, } \\
\text { Ars generalis } \\
\text { ultima } \\
(1305-08)\end{array}$ & $\begin{array}{l}\text { Donato, } \\
\text { Ars minor e } \\
\text { Ars Maior } \\
\text { (sec. IV) }\end{array}$ & $\begin{array}{l}\text { Prisciano, } \\
\text { Institutiones } \\
\text { grammaticae } \\
\text { (sec. V-VI) }\end{array}$ & $\begin{array}{l}\text { Isidoro } \\
\text { di Siviglia, } \\
\text { Etymol. } \\
\text { (sec. VII) }\end{array}$ & $\begin{array}{l}\text { Thomas } \\
\text { of Erfurt, } \\
\text { Grammatica } \\
\text { speculativa } \\
(1300-1310)\end{array}$ \\
\hline Nomen & Nomen & Nomen & Nomen & Nomen \\
\hline Pronomen & Pronomen & Verbum & Pronomen & Pronomen \\
\hline Verbum & Verbum & Participium & Verbum & Verbum \\
\hline Participium & Aduerbium & Pronomen & Adverbium & Participium \\
\hline Coniunctio & Participium & Praepositio & Participium & Adverbium \\
\hline Praepositio & Coniunctio & Adverbium & Coniunctio & Coniunctio \\
\hline Adverbium $^{33}$ & Praepositio & Interiectio & Praepositio & Praepositio \\
\hline Interiectio & Interiectio & Coniunctio & Interiectio & Interiectio \\
\hline
\end{tabular}

La rispondenza s'interrompe quando si procede all'esposizione delle singole voci. Il nome corrisponde ai principi dell'Arte (bontà, grandezza, ecc.), così come il pronome, in quanto "pro proprio nomine ponitur». ${ }^{33}$ Llull insiste sul fatto che i nomi (principi) dell'Arte sono applicabili a tutte le scienze, e non viceversa, perciò sono generali.

Tutte le parti dell'orazione sono ricondotte alla regola $\mathrm{C}$, tranne l'interiezione, che viene collegata alla voluntas, secondo una definizione canonica. Al verbo, al participio, alla congiunzione e all'avverbio si applica, in particolare, la seconda specie della regola C. Questo spiega perché viene anticipata la posizione dell'avverbio rispetto allo schema iniziale. La seconda Regola dell'Arte $(\mathrm{C})$ riguarda la quidditas e permette di rispondere alla domanda «che cos'è?». $\mathrm{Si}$ articola in quattro specie, che sono così definite nell'Ars brevis:

'Quid' habet quattuor species. Prima est definitiua; sicut quando quaeritur: Quid est intellectus? Et respondendum est, quod ipse est illa potentia, cui proprie competit intelligere. Secunda species est, quando quaeritur: Intellectus quid habet in se coessentialiter? Et respondendum est, quod habet sua correlatiua, scilicet intellectiuum, intelligibile et intelligere; sine quibus esse non potest; et etiam sine ipsis esset otiosus et indigens natura, fine et quiete.

Tertia species est, quando quaeritur: Quid est ens in alio? Sicut quando quaeritur: Quid est intellectus in alio? Et respondendum est, quod est bonus, intelligens in bonitate; et magnus intelligens in magnitudine, etc.; et in grammatica grammaticus, et in logica logicus; et in rhetorica rhetoricus, etc.

Quarta species est, quando quaeritur: Quid habet ens in alio? Vt cum dicitur: Quid habet intellectus in alio? Et est respondendum, quod in scientia intelligere, et in fide credere. ${ }^{34}$

33. Sulla complessità del rapporto fra nome e pronome nella grammatica speculativa, cfr. Costantino Marmo, Semiotica e linguaggio nella scolastica: Parigi, Bologna, Erfurt 1270-1330: la semiotica dei Modisti, Roma: Istituto Storico Italiano per il Medio Evo, 1994, p. $160-174$.

34. ROL XII, 1984, p. 214. 
Nella seconda specie si colloca la dottrina dei correlativi, fondamentale per comprendere l'articolazione dell'ontologia lulliana, nella quale essere e attività sono inseparabili. Llull dota questa attività di una struttura generale e sempre uguale a un'espressione linguistica corrispondente. I termini correlativi rappresentano la declinazione della sostanza nei componenti potenziali e attuali di ogni essere: le parti attive sono indicate dal suffisso -tivum, le passive da -bile, e la loro unione dal verbo in -are. A partire dalla Lectura super figuras Artis demonstrativa (1285-7) tale grammatica ontologica si estende da Dio a tutti i livelli della realtà, e ciò permette a Llull di strutturare la visione trinitaria del mondo. Risulterà ora più chiara l'applicazione della seconda specie della Regola C alle singole parti del discorso, a cominciare dal verbo. Per esempio, magnificare e bonificare, con riferimento ai principi dell'Arte, indicano un atto "quemadmodum et verbum». Alla stessa specie si riconducono il participio (bonificans e bonificatus, ecc.), e la coniunctio: come l'infinito unisce attività (-ans) e passività (-atum) in un composto perfetto (-are), così (sicut) la congiunzione unisce diverse parole (dictiones) per formare un'orazione completa (perfectam orationem). L'avverbio ricade nella medesima specie della regola C quale scopo dell'agens che vuole produrre il proprio effetto. Alla quarta specie della regola $\mathrm{C}$ si ricorre per la preposizione, di cui il grammatico si serve per ridurre il caso (in senso grammaticale) all'atto. ${ }^{35}$

Terminata l'esposizione delle parti del discorso, l'applicazione continua con gli altri modi elencati: i casi, la coniugazione, la declinazione, il genere, il regimen, la costruzione, l'ortografia e la figura. Per i casi il riferimento principale è quello alla seconda specie della regola $\mathrm{C}$, cioè alla relazione correlativa che essi esprimono in forma diversa: come il bonificans è "per se est non descendens ab aliquo alio» ed è principio di bonificatum e bonificare, così il nominativo è principio degli altri casi; il genitivo discende dal proprio nominativo come bonificatum e bonificare derivano dal bonificans; lo stesso vale per il dativo e l'ablativo, che trovano la loro collocazione nella declinazione correlativa. Al genitivo si può applicare anche la terza specie della regola $\mathrm{D}$, che indica il possesso («di chi o di che cosa è»). L'accusativo è implicito nella terza specie della regola $\mathrm{C}$, in quanto fine degli atti dell'agente, mentre il vocativo è ricondotto alla quarta specie («in ipsa namque vocans habet suum vocatus obiective») e alla regola B (Utrum?). Anche la coniugazione, la declinazione e il genere sono ricondotti alla seconda specie della Regola C: come la bonitas è principio e causa dei suoi correlativi, che da essa discendono e che si differenziano secondo le loro diverse proprietà, così (sicut) nella coniugazione si ha un

35. Nel Llibre de contemplació si legge un passo che richiama il parallelo tra parti del discorso e trinità (ORL VIII, 1914, p. 151-152): «paraula està en uniò e en .iij. coses: nom e verb e preposició, e assò metex se seguex de vou qui no puría esser sens moviment e sense àer e sens oyment (...). On, com totes estes coses, e axí de les altres coses semblants a aquestes, no poguessen esser en esser sens que cascuna cosa no fos en .iij. coses, per assò es significat al humà enteniment que l'acabament del creador qui ha creades les coses en unió e en triplicitat, sia en unió e en trinitat». 
principio dal quale derivano molte voci, che si differenziano reciprocamente per persona, tempi e modi, ecc.; per la declinazione si afferma che, come bonificatus e bonificare discendono dal bonificante per generazione (generando), così (sicut) nella coniugazione si ottiene un caso dall'altro. Per il genere si ricorda che bonificans significa il genere maschile in quanto azione, bonificata quello femminile in quanto passione, bonificare il neutro. ${ }^{36}$

Ai modi riguardanti la sintassi, cioè il regimen e la costruzione, si applicano invece i principi relativi. ${ }^{37}$ Il regimen è ricondotto al terzo triangolo della figura $T$ (maggiore, minore, uguale), implicito nella maggiore potestà e virtù del reggente rispetto al suo subordinato. Qui Llull introduce un interessante, ma non originale, ${ }^{38}$ paragone di natura politica, ricordando che governare spetta a chi possiede maggiore potenza e virtù. La costruzione fa riferimento al secondo triangolo della figura $\mathrm{T}$ (principio, medio, fine): come il principio precede il medio e il fine, così (sic quidem et) nella costruzione il suppositum precede e l'appositum segue. I termini suppositum e appositum ricorrono soltanto in questo passo. Nei testi della seconda metà del XII secolo questa coppia di parole, con i relativi verbi, si era fissata in ambito grammaticale, mentre era divenuta specifica della logica quella subiectum/obiectum, che lo stesso Llull adotta nella relativa applicazione dell' $A G U .{ }^{39}$

L'ortografia è implicata nella Regola K o della modalità, che riguarda l'articolazione delle parti all'interno di un tutto e la semblança che l'insieme trasmette fuori di sé. L'ortografia insegna infatti ad unire lettere, sillabe,

36. Sui generi, cfr. anche la Rhetorica nova (ROL XXX, 2005, p. 63-64).

37. Sulle accezioni della parola regimen, cfr. la ricostruzione di Paola BENEDinI, «La teoria sintattica dei Modisti: attualità dei concetti di reggenza e dipendenza», Lingua e stile, n. 23, 1988, p. 113-135, a p. 120, n. 2. Secondo Geoffrey BursilL-Hall (Speculative grammars, cit., p. 63-64) i modisti non ricorrono a questo vocabolo preferendo, in generale, determinare e determinatio.

38. Cfr. Siger de Courtrai, Les oeuvres de Siger de Courtrai, étude critique et textes inédits par Gaston Wallerand, Louvain: Institut supérieur de philosophie de l'Université, 1913, p. 139: "Circa tertium est intelligendum quod regimen in entibus gramaticalibus debet sumi ad similitudinem regiminis veri. Nunc secundum quod patet primo Politicorum, in qualibet multitudine digniora regunt minus digna. Ideo, ut patet ex octavo Physicorum et duodecimo Metaphysice, in genere entium est devenire ad aliquod primum dignissimum et nobilissimum, quod omnia entia regit, videlicet causa prim a vel Deus, ut patet ex libro de Causis. [...] Nunc autem ad similitudinem huius sumitur regimen inter partes orationis, ita quod est devenire ad aliquam partem orationis dignissimam respectu talis multitudinis, quia multitudinem et orationem complet, quae proprie regit omnes alias partes et a nulla alia regitur, videlicet verbum».

39. Sulla storia di questa terminologia, cfr. Thurot, Extraits de divers manuscrits latins, cit., p. 217-218 e 265; Irène Rosier, «Lintroduction de notions de sujet et prédicat dans la grammaire médiévale», Archives et documents de la Société d'Histoire et d'Epistemologie des Sciences du Langage (SHESL), n. 10, 1994, p. 81-119; Lola BADIA, "A propòsit de Ramon Llull», cit., p. 191-192. Sulle accezioni che nei grammatici speculativi assumono suppositum/appositum, cfr. Karen Margareta Fredborg, "Speculative Grammar», in Peter Dronke (a cura di), A History of Twelft-Century Western Philosophy, Cambridge, Cambridge University Press, 1988, p. 177-195, a p. 187-195. 
parole, frasi, accenti ecc. ed estende il proprio dominio dalle lettere alla prosodia. ${ }^{40}$

La trattazione si chiude con la figura, implicata nel primo triangolo della Figura T (differenza, concordanza, contrarietà), cui si applica la definizione di contrarietà quando è un vitium che va contro la norma, o quella di concordanza quando è motivata. ${ }^{41}$ Nelle opere di Donato e di Prisciano la figura veniva trattata all'interno delle parti del discorso in rapporto alla composizione. I modisti interpreteranno il fenomeno in termini semantici, come significato o assenza di significato delle parti che compongono una parola. ${ }^{42}$ Secondo Llull la figura deve avere una ratio, altrimenti è da scartare e da considerare un vizio (è sinonimo di improprietas). L'ultimo modo della classificazione lulliana ci conduce nel dominio della retorica. Come osserva Josep Lluís Martos: "Gramática y retórica funden sus límites en la Edad Media a partir del concepto de transgresión gramatical». ${ }^{43}$ Tale sovrapposizione si deve al fatto che il dominio della grammatica contemplava tanto il rispetto delle norme quanto la loro violazione, considerata ora un vitium, ora una licentia poetarum. La soluzione di tali vizi avveniva nelle figure o schemata (termine quest'ultimo usato da Donato e da Isidoro di Siviglia). Nelle arti del trivio il processo di reciproca influenza è circolare: va dalla retorica alla logica e dalla logica alla grammatica. A conferma di questa circolarità, si può osservare che il termine figura per indicare i tropi non compare nella Rhetorica nova, mentre si trovano le figurae dictionis (fallacie) nella Logica d'Algatzel, opera che Llull aveva tradotto in prosa latina e poi ridotto in versi catalani all'inizio del suo percorso (1271-2?). La stessa fluidità si può osservare nella trattazione della constructio, che Llull sviluppa nella retorica in rapporto all'ordo piuttosto che nei sintetici profili riservati alla grammatica.

\section{Tra grammatica e filosofia del linguaggio}

Le considerazioni che si possono trarre dall'analisi dettagliata delle occorrenze investono aspetti diversi della produzione lulliana. La grammatica non sembra interessare Llull, se non nella veste di disciplina propedeutica o come proiezione utopica di una unità linguistica dei popoli, almeno fino all' $A G U$. Le omissioni confermano una scarsa attenzione verso questa disciplina, che resta l'unica arte del trivio priva di una trattazione specifica, paragonabile a quella svolta nella Rhetorica nova (1301) e nella Logica nova (1303). Abbiamo visto che nell'Aplicació de l'Art general (1301) Llull affronta la logica e la retorica ma omette la grammatica. Nella Lectura la grammatica viene solo citata, men-

40. Cfr. Badia, «A propòsit de Ramon Llull», cit., p. 191.

41. Di questa distinzione si trovano vari esempi in Thurot, Extraits de divers manuscrits latins, cit., p. 458 e ssg.

42. Cfr. Bursill-Hall, Speculative grammars, cit., p. 59-60.

43. Josep Lluís Martos, «La gramatización de la poética en la Edad Media: crisis y prescripción», Romace Philology, n. 61, Fall 2007, p. 125-146, a p. 127. 
tre i temi della costruzione e dei rapporti di nobiltà tra le parti del discorso, che avrebbero potuto trovare accoglienza nella sintassi, sono sviluppati all'interno della retorica.

Il metavocabolario lulliano non si allontana da quello corrente. L'adozione di un lessico comune, riscontrato nell'analisi delle definizioni, appare scontata, poiché l'applicazione si rivolge a chi conosce la grammatica normativa del latino - da qui la parsimonia nella descrizione delle parti del discorso - e all'adepto che ha già appreso il meccanismo dell'Arte. La trattazione si concentra infatti sull'analogia tra le due, senza indugiare sulle accezioni dei modi. Anche per questa ragione la ricerca delle fonti su base lessicale risulta poco efficace, né serve a individuare una possibile influenza della grammatica speculativa, in voga sul finire del XIII sec., dal momento che anche i modisti adottano la terminologia tradizionale dotandola di un significato tecnico. La parola modus nell' $A G U$ ritaglia concetti convenzionali, non riferibili ai modi significandi, essendi ed intelligendi nel valore che avevano assunto nella grammatica speculativa. ${ }^{44}$ Se una suggestione esiste, l'esito è la sua riduzione all'ontologia della serie correlativa, nella quale i modi essendi e modi intelligendi coincidono.

I lavori che ipotizzano un'influenza modista hanno come testo di riferimento, più che l'AGU, la Rhetorica nova. La maggiore e programmatica trasparenza delle fonti, la parsimonia dei rinvii artistici e lo sconfinamento nella logica rendono quest'opera più disponibile all'esercizio delle corrispondenze. ${ }^{45}$ I curatori della Retòrica nova della TORL osservano che la relazione tra bellezza ontologica e bellezza dell'ornato comporta una distinzione filosofica tra essenza e accidente, tra forma e materia che non ha riscontri nella grammatica normativa, mentre evidenzia una affinità con quella speculativa. ${ }^{46} \mathrm{Nel}$ commento al testo si offrono alcuni riscontri lessicali, soprattutto con la Grammatica speculativa di Tommaso di Erfurt, che riguardano: il concetto di materia e forma applicato ai verba; ${ }^{47}$ il nesso tra bellezza del dettato e verità, che potrebbe connettersi alla «doctrina dels lògics modistes, que pressuposaven que els diversos modes que revestia la parla (modi loquendi) no eren arbitraris, sinó que reflectien veritablement els diversos modes d'ésser de les coses (modi

44. Lola BADIA, "A propòsit de Ramon Llull», cit., p. 192, sostiene che il termine sia da interpretare come un'esibizione di una «marca supeficial de competència tècnica».

45. Cfr. Elena Pistolesi, «Retorica, lingue e traduzione nell'opera di Ramon Llull», in M. I. Ripoll e M. Tortella (a cura di), Ramon Llull i el Lul.lisme: pensament i llenguatge. Actes de les jornades en homenatge a J. N. Hillgarth i A. Bonner, Barcelona-Palma de Mallorca: Universitat de Barcelona-Universitat de les Illes Balears, 2012, p. 313-327 (Col-lecció Blaquerna, 10).

46. TORL 1, 2006, p. 53: «Per a un modista "dictio est vox significativa”. Llull hauria pogut acceptar aquesta formulació i, casant-la amb l'ontologia inherent a l'Art, aproximar-se a la retòrica per aquesta via insòlita».

47. Ibid., p. 99, n. 10: «Els gramàtics modistes se servien de la contraposició aristotèlica matèriaforma per generar oposicions binàries que aplicaven en anàlisis diverses», con rinvio a G. L. Bursill-Hall, Speculative Grammars, cit., p. 50-54 e a Tommaso di Erfurt, Grammatica Speculativa, 24 «Modus intelligendi materiam» (ed. Bursill-Hall, p. 208). 
essendi). En termes de lingüística medieval, els modistes atribuïen als noms una suppositio naturalis", concetto di cui si riporta la definizione tratta dal Tractatus di Pietro Ispano. ${ }^{48}$ Mauricio Beuchot ritiene che questi riscontri dimostrino il rapporto tra Llull e la grammatica speculativa. ${ }^{49}$ L'esempio più significativo di tale influenza sarebbe dato dalla tripartizione in forma, materia e fine dei verba, che già Mark D. Jonhston aveva discusso anche in rapporto ai grammatici speculativi, concludendo che «it is tempting to imagine that Llull's accounts reproduce their efforts. His results remain, nonetheless, far less systematic than those of the modistae. Ultimately, it is easiest to understand the tripartite hylemorphic scheme from the Rethorica nova as a kind of distinctio». 50

Per andare oltre il riscontro lessicale, si dovrà spiegare come avviene l'articolazione dei modi, un punto problematico che investe l'isomorfia tra l'ordine del pensiero e la struttura del linguaggio, così formulato da Maierù in riferimento ai modisti:

E tuttavia c'è un'evidente tensione tra la definizione delle parti del discorso, fornita dai nostri maestri, che ne mette in luce le rispettive funzioni, e l'ordine della realtà al quale essi stessi si richiamano. Ma la tensione è risolta, almeno tendenzialmente, nel senso dell'affermazione dell'autonomia dell'intelletto e di conseguenza dell'autonomia della grammatica. ${ }^{51}$

In Llull non si trova, né si potrebbe trovare dati i presupposti stessi dell'Arte, alcuna rivendicazione sull'autonomia della grammatica, che per i modisti è una scienza in senso aristotelico, e speculativa in quanto dotata di un proprio statuto epistemologico. Llull non ricorre alla teoria della suppositio, che domina la semantica terminista all'inizio del sec. xIV, menzionata dai commentatori della Retòrica nova, come soluzione al problema della referenza, ${ }^{52}$ poiché il suo sistema opera direttamente nel mondo reale platonico. Per questa ragio-

48. Ibid., p. 115 , n. 30. Ancora, per la frase lulliana «vox enim est subiectum, in quo verba ponuntur", si ricorda che per i logici e per i grammatici medievali la vox era oggetto di studio in quantum signum, con un rinvio a Tommaso di Erfurt e Guglielmo di Ockham (ibid., p. 179, n. 99).

49. Per Beuchot, «Some Features of the Semantics», cit., p. 112, n. 2: «The connection between Llull and the linguistic tradition of speculative grammar and the modistae, is demonstrated by Josep Batalla, Lluís Cabré and Marcel Ortín, in [11, p. 99, note 10 and p. 101, note 13]». La nota di p. 101 citata come riscontro recita: «Les «diccions» (dictiones) són «la matèria de les paraules» (materia verborum sunt dictiones); cf. \$142. Una «dicció» és, doncs, el signe oral o escrit del significat d'una paraula. Cf. $\$ 169$, on es parla de "pronunciar diccions» (dictiones proferre); Llibre de contemplació, 3.155.19 (OE II, p. 448): «Hom escriu paraula en diccions, per tal que ligent entena hom los significats que paraules demostren». Compareu la manera aristotèlica de definir-les de Tomás d'Erfurt, Grammatica especulativa, 6.11 (ed. Bursill-Hall, p. 148): «dictio est vox significativa».

50. Mark D. Johnston, The Evangelical Rhetoric of Ramon Llull. Lay Learning and Piety in the Christian West around 1300, Oxford: Oxford University Press, 1996, p. 43.

51. Alfonso Maierù, «La grammatica speculativa», cit., p. 156.

52. Cfr. Costantino Marmo, La semiotica del XIII secolo, cit., p. 29-49. 
ne si interessa solo al modo in cui una cosa ne significa un'altra, in cui il creato significa la realtà divina, senza entrare nel merito dell'articolazione tra segno e referente. La conferma si trova nel Liber de significatione (1304), privo di considerazioni linguistiche, dedicato esclusivamente alle relazioni tra principia e creature secondo un rapporto di significazione che Llull designa con nomi diversi, quali fra i quali manifestació e revelació. ${ }^{53}$

Nell'opera Lo sisèn seny, lo qual apelam affatus (1294) il tema dell'espressione vocale è risolto nella connessione naturale e materiale tra concetto e nuovo senso; ${ }^{54}$ nella Rhetorica nova la bellezza del dettato dipende esclusivamente dalla natura del referente e dal posto che occupa all'interno di una specifica gerarchia, che può essere entitativa, etica, ecc., ma non intrinsecamente linguistica. Tale posizione, eterodossa rispetto al percorso disegnato dalla tradizione aristotelica, sarà rivisitata nelle opere spurie come la Logica brevis, nella quale fa la sua comparsa la suppositio, ${ }^{55}$ e il Liber de universalibus, trasmesso da due mss. latini della metà del sec. XIV, che incorpora i modi significandi nella definizione della grammatica:

6.9 De grammatica. Congruitas et rectitudo loquendi est subiectum in grammatica. Finis recte loquendi, pronuntiare et scribere. Principia sunt: Littera, syllaba, dictio, oratio uel octo partes orationis aut concordantia substantiui et adiectiui, suppositi et appositi, relatiui et antecedentis, uel etiam modi significandi. ${ }^{56}$

L'ordine della realtà al quale Llull riconduce i modi della grammatica nell' $A G U$ è fondato sull'analogia (sicut) con le regole dell'Arte e, in primo luogo, con i correlativi della seconda specie della regola $\mathrm{C}$, dalla quale dipendono i casi, le parti dell'orazione e i verbi, con le eccezioni della sintassi, ricondotta al triangolo inizio-medio-fine, e della figura interpretata alla luce dei principi di concordanza e contrarietà.

L'Arte non solo è capace di dimostrare i principi delle scienze particolari in quanto universale, ma anche di individuarli e plasmarli, secondo l'accezione tutta lulliana di invenire. ${ }^{57}$ Tale ricerca nei riguardi della grammatica trova il suo fondamento nel declinari, termine che accomuna i nove modi all'attività dell'essere. Come i nomi (vocabula nominalia) sono compresi nelle cinque declinazioni e si possono plasmare secondo regole costanti, così «suo

53. Riprendo qui le considerazioni svolte da Bonner, Art and Logic, cit., 198-200.

54. Cfr. Elena Pistolesi, "Paraula és imatge de semblança de pensa”. Origine, natura e sviluppo dell'affatus lulliano», $S L$, n. 36, 1996, p. 3-46.

55. Mauricio Bеuснот, «Some Features of the Semantics», cit., ricostruisce la convergenza tra logica dei termini e grammatica nel lullismo, sottolineando più volte che negli scritti originali del filosofo non si trova alcun accenno alle proprietà logiche dei termini, ma sembra accantonare questa premessa fondamentale nel corso dell'esposizione. Per esempio, il paragrafo «Syntax and Semantics in Llull» (p. 108-111) espone solo le tesi lullistiche, insistendo sulla suppositio, estranea al pensiero di Llull.

56. ROL XII, 1984, p. 167-168.

57. Ruiz Simon, L'Art de Ramon Llull, cit., p. 405-416. 
modo", cioè fatte le debite distinzioni, tutte le quaestiones si possono ridurre alle dieci dell'Arte. ${ }^{58}$ Declinare è parola usata nel Compendium seu Commentum Artis demonstrativae (1289), ${ }^{59}$ nell'Art amativa (1290), nella Taula general (1293-4) e nell'Arbre de filosofia desiderat (1294) in rapporto alla dottrina dei correlativi.

La marginalità della grammatica disegnata fin qui contrasta con l'interpretazione di un Llull simpatizzante della corrente speculativa. Tale orientamento si fa risalire di solito a Platzeck, i cui lavori sono anteriori alla cospicua letteratura dedicata ai modisti a partire dagli anni Settanta. ${ }^{60}$ Con 'grammatica speculativa' egli intende una grammatica ontologicamente fondata, con riferimento precipuo alla dottrina stoica e al platonismo nelle sue sfumature medievali - in particolare nella veste che assunse presso la scuola di Chartres ${ }^{61}$ - , contrapposta alla grammatica positiva o descrittiva. ${ }^{62}$

Per comprendere lo svolgimento della grammatica nell' $A G U$ non è necessario attingere ai modi tecnicamente intesi perché si può spiegare entro i fondamenti dell'Arte. Non c'è traccia nell'opera di Llull di doppia articolazione, di rivendicazioni forti relative all'autonomia della grammatica come scienza, né di definizioni delle parti del discorso che rinviino alla complessa costruzio-

58. Tale affermazione si legge nel prologo dell' $A G U$ (ROL XIV, 1986, p. 26), segnalata a suo tempo da Tomás Carreras i Artau, "El Llenguatge filosòfic de Ramon Llull», in Homenatge a Antoni Rubió $i$ Lluch 1, Barcelona, 1936, p. 545-552: «Regulae sunt decem, scilicet: Vtrum, quid, etc., ut in alphabeto iam significatum est. Istae regulae sunt decem quaestiones generales, per quas oportet esse omne quaesitum. Et quando id, de quo quaeritur, ponitur in ipsis, est lucefactum et coloratum, et etiam significatum intellectui secundum essentiam et naturam regulae, sicut patet in grammatica. Nam sicut omnia uocabula nominalia declinabilia includuntur in quinque declinationibus, et possunt declinari per ipsas, sic suo modo omnes aliae quaestiones praeter istas huius Artis, quae fieri possunt, includuntur in ipsis decem, et etiam ad ipsas quidem reducuntur; et etiam per ipsas regulantur ratione generalitatis, quam habent».

59. Chi vuole apprendere l'Arte dovrà «declinare namque terminos figurarum dicendo sub condicionibus bonitatis, bonificativum, bonificabile bonificare bonificatum (ecc.)»; pur ammettendo che non è un modo di parlare abituale per chi usa il latino, «hunc autem de vi et virtute terminorum hujus Artis sic declinari oportet» (MOG III, 1722, Int. vI, p. 160).

60. Erhard-Wolfram Platzeck, «La combinatoria luliana. Un nuevo ensayo de exposición e interpretación de la misma a la luz de la filosofía general europea», Revista de filosofía, n. 12-13, 1953-1954, p. 575-609 e 125-165; ID., «La Figura T del Arte luliana y la doctrina de las significaciones», Studia monographica et recensiones, n. 9-10, 1953-1954, p. 35-49.

61. Gli autori citati come fonti sono infatti Bernardo di Chartres, Yves di Chartres, Teodorico di Chartres, Guglielmo di Conches, Gilberto Porretano e Giovanni di Salisbury.

62. L'ottica della sua indagine è riassunta nella frase posta in corsivo nell'originale (PLATzecK, "La Figura T del Arte luliana», cit., p. 48): «Todo lo dicho aparece como una especie de filosofía de la gramática; pero es en verdad una teoría general de las más abstractas significaciones del espíritu humano en sus relaciones mútuas». Platzeck valorizza la figura T in quanto figura significationum e il ricorso ai gradi dell'aggettivo come corrispettivo della declinazione della sostanza, riassunti nel triangolo majoritat-igualtat-minoritat. Sui gradi dell'aggettivo, cfr. M. D. Johnston, The Evangelical Rhetoric, cit., p. 105 e n. 26, che non li considera la base per una nuova teoria della dimostrazione, come sostenuto da Platzeck, né un omaggio alla grammatica speculativa. 
ne della dottrina modista. Questo non esclude una conoscenza dei testi o un loro riecheggiamento, ma ciò che distingue Llull dagli esponenti di questa corrente è prima di tutto la finalità. Ai seguaci di una concezione aristotelica della scienza, da cui discende anche l'autonomia della grammatica, Llull contrappone i principi generali, comuni e fondanti di ogni sapere. Le affinità si possono stabilire, eventualmente, tra il «realismo moderato" adottato da alcuni esponenti della corrente modista e la progressiva ontologizzazione del metodo artistico. La distanza più evidente riguarda l'articolazione dei piani che conducono fino all'espressione vocale e alle parti del discorso. Le questioni poste sul terreno della grammatica confermano che quella lulliana è una filosofia del linguaggio "dispersa», ${ }^{63}$ che risponde a questioni puntuali, la cui coerenza va ricercata nel disegno complessivo dell'Arte e nella sua evoluzione. Le tracce di questa filosofia sono numerose, dall'allusione alla tesi della convenzionalità del segno, ${ }^{64}$ alla dottrina del verbum (distinto in sensibile e intellettuale in rapporto al contenuto del conceptum, non alla vox), ${ }^{65}$ fino alla scoperta dell'affatus. Il sesto senso, che sente "la manifestació de la concepció» trasformandola in suono, sviluppa il tema della comunicazione in termini psicologici, non semiotici. Esso rappresenta il superamento del segno, perché riduce il linguaggio entro la sfera materiale della sensibilità. Nella Rhetorica nova si privilegia l'espressione della verità ricorrendo alla commistione, non del tutto riuscita, tra la retorica tradizionale e la gerarchia entitativa. Il Liber de refugio intellectus contiene un accenno alla polisemia delle parole, ma - come sottolinea Bonner - tale rilievo isolato non è solo semantico, è semantico e formale insieme. ${ }^{66} \mathrm{I}$ riferimenti alle questioni linguistiche, sia collegate alla predicazione e alle traduzioni sia teoriche, si potrebbero moltiplicare ma, nel complesso, questi aspetti non sono oggetto di una trattazione sistematica. L'unico passo in cui affatus e grammatica sono citati insieme si legge nell'Arbre de ciència: "demanà l'ermità si gramàtica fo enans considerada per necessitat de effatus que per la sua retòrica. - Sol. Gran prou fora en lo món si la gramatica dels latins sabessen totes les nacions». ${ }^{67}$ La risposta non chiarisce affatto la natura del loro vincolo, né ci soccorre in questo la Rhetorica nova, dove non si menzionano né il sesto senso né il ruolo della grammatica. L'elemento unificante è dato dal riferimento di ciascuno di questi ambiti (psicologico, conoscitivo, pragmatico) al percorso dell'Arte. La grammatica è un altro capitolo della dispersa filosofia del linguaggio che Llull non si preoccupa di convogliare in una speculazione organica e stringente come quella dei modisti. La sin-

63. Per un quadro complessivo della filosofia del linguaggio lulliana, cfr. Sebastià TRIAS MerCANT, Ramon Llull. El pensamiento y la palabra, Palma de Mallorca: El Tall, 1993; Joan Tusquets i Terrats, La filosofia del llenguatge en Ramon Llull, pr. Miquel Arbona, Barcelona: Editorial Balmes, 1993.

64. Liber de praedicatione, ROL IV, 1963, p. 409.

65. Quaestiones per Artem demonstrativam seu inventam solubiles (1289), MOG IV, 1729, Int. III, p. 76, Q. LIV «Quomodo anima generet Verbum?».

66. Bonner, Art and Logic, cit., p. 237.

67. ORL XIII, 1926, p. 250. 
tesi estrema di quanto abbiamo detto potrebbe essere il capovolgimento del titolo di questo contributo: «la grammatica lulliana dall'Arte al trivium».

Tavola delle definizioni di grammatica

\begin{tabular}{lll}
\hline Opera & Definizione & Contesto \\
\hline 1. II.A.6. Doctrina & [1] Gramatica es dretament par- & De les.VII. Arts \\
pueril (1274-6) & lar e escriure, e per assó es aleta \\
(NEORL VII, & a esser comú lenguatge a les \\
pp. 189-190) & gents qui per luyetat de terres e \\
& de participació son desvariables \\
& en lur lenguatge. \\
& [2] Fil, si vols apendre gramatica \\
& .iii. cozes te convenen a saber: \\
& costrucció, declinació e vocables \\
& [...] \\
& [3] Cor aurás apresa gramatica \\
& en est libre, enaprés aprin-la en \\
& lo Libre de defenicions e de que- \\
& stions per so que anans ages les \\
& altres sciencies. Si en nula art ne \\
& sciencia vols entrar, primera- \\
& ment te cové a passar per esta art \\
& de gramatica, qui es portal per lo \\
& qual hom passa a saber les altres \\
& sciencies.
\end{tabular}




\begin{tabular}{|c|c|c|}
\hline Opera & Definizione & Contesto \\
\hline $\begin{array}{l}\text { 2. III.11 } \\
\text { Taula general } \\
\text { (Tunisi-Napoli } \\
\text { 9/1293-1/1294) } \\
\text { (ORL XVI, } \\
\text { pp. 490-491) }\end{array}$ & $\begin{array}{l}\text { Aquesta sciencia es general a } \\
\text { totes siencies per so cor es de ge- } \\
\text { nerals comensamens e per so cor } \\
\text { les altres sciensies an comensa- } \\
\text { mens especials, axí com theolo- } \\
\text { gia qui à especials comensamens, } \\
\text { so es assaber, fe esperansa e cari- } \\
\text { tat, e filosofia, forma e materia e } \\
\text { privació, e dret, jutje e justicia, e } \\
\text { medisina, metge e sanitat, e axí } \\
\text { com justicia prudensia fortitudo } \\
\text { atrempansa qui son comensa- } \\
\text { mens de moralitat, e congruitat } \\
\text { e dretament parlar qui son co- } \\
\text { mensamens de gramàtica, e veri- } \\
\text { tat e falsetat, de lògica, e orde e } \\
\text { bellea de paraules qui son co- } \\
\text { mensamens de rectòrica, e music } \\
\text { e veu, de musica, e arismètic e } \\
\text { nombre, de arismètica, e je- } \\
\text { omètric e mesures, de geomètri- } \\
\text { ca, e astròlog e signes e planetes, } \\
\text { de estrologia, e mecànic e estru- } \\
\text { mens e figures qui son comensa- } \\
\text { mens de art mecànica. Totz } \\
\text { aquestz [...] }\end{array}$ & $\begin{array}{l}\text { De les questions de les Regles } \\
\text { Questió k. Còm es aquesta } \\
\text { sciencia general a totes scien- } \\
\text { cies? [...] Totz aquestz comen- } \\
\text { samens son enplegats en los } \\
\text { comensamens d'aquesta art, } \\
\text { quar totz son bons e grans, e } \\
\text { los altres; e per asò aquesta } \\
\text { art es general a aquells ab sos } \\
\text { comensamens generals, e } \\
\text { enclina.s a les altres sciencies } \\
\text { segons que.ls comensamens } \\
\text { d'aquelles estan en los seus } \\
\text { comensamens enplegats, e } \\
\text { està desús a aquelles, enaxí } \\
\text { com genre qui està sobre } \\
\text { especia, e usa dels comensa- } \\
\text { mens d'aquelles sciencies se- } \\
\text { gons l'orde e l'ús que à de sos } \\
\text { propis comensamens, la } \\
\text { pràtica del qual ús es donada } \\
\text { en esta sciencia. }\end{array}$ \\
\hline $\begin{array}{l}\text { 3. III.23 Arbre de } \\
\text { ciència (Roma } \\
\text { 29/9/1295- } \\
\text { 1/4/1296) } \\
\text { (ORL XI, } \\
\text { pp. 215-216) }\end{array}$ & $\begin{array}{l}\text { Gramatica es art qui ensenya a } \\
\text { parlar ordonandament, e posar } \\
\text { accents en los vocables segons } \\
\text { ques cové, e concordar los verbs } \\
\text { els noms e les preposicions, els } \\
\text { substantius els adjectius, e les } \\
\text { altres parts de la art. E aquest } \\
\text { ordre e procés comença prime- } \\
\text { rament en les formes primeres } \\
\text { sembrades en los arbres en qui } \\
\text { son ordonades segons cors natu- } \\
\text { ral, del qual orde ha lo gramàtic } \\
\text { estinct natural don trau lorde } \\
\text { del hàbit artificial en parlar e en } \\
\text { fer bon latí, e en esquivar fals } \\
\text { accent, en lo qual latí pos los si- } \\
\text { gnificats dels vocables qui son en } \\
\text { vulgar. }\end{array}$ & $\begin{array}{l}\text { V. De LES FULLES DE L'ARBRE } \\
\text { HUMANAL } \\
\text { 6. Dels habits del Arbre } \\
\text { humanal: } h \text { ) De gramàtica }\end{array}$ \\
\hline
\end{tabular}




\begin{tabular}{|c|c|c|}
\hline Opera & Definizione & Contesto \\
\hline $\begin{array}{l}\text { 4. III.64.bis } \\
\text { Lectura Artis } \\
\text { quae intitulatur } \\
\text { Brevis practica } \\
\text { Tabulae generalis } \\
\text { (Genova } \\
1 / 2 / 1304 \text { ) } \\
\text { (ROL XX, } \\
\text { p. } 397 \text { ) }\end{array}$ & $\begin{array}{l}\text { Grammatica siquidem consi- } \\
\text { derat in sermone congruum et } \\
\text { incongruum. }\end{array}$ & $\begin{array}{l}\text { Diuiditur autem philosophia } \\
\text { in tres partes: Prima pars est } \\
\text { de triuio, in quo agitur de } \\
\text { sermone; secunda de quadri- } \\
\text { uio, in quo agitur de ente } \\
\text { mathematico; et in tertia } \\
\text { parte agitur de ente naturali. } \\
\text { Triuium autem constituunt } \\
\text { tres scientiae de sermone, } \\
\text { scilicet, grammatica, dialec- } \\
\text { tica et rhetorica. }\end{array}$ \\
\hline $\begin{array}{l}\text { 5. III.80 Ars } \\
\text { generalis ultima } \\
\text { (Lione/Pisa } \\
11 / 1305- \\
3 / 1308 \text { ) } \\
\text { (ROL XIV, } \\
\text { pp. 367-370) }\end{array}$ & $\begin{array}{l}\text { 88. De grammatica. Gramma- } \\
\text { tica est ars ad inueniendum } \\
\text { modum recte loquendi recteque } \\
\text { scribendi. } \\
\text { Per definitionem differentiae } \\
\text { grammatica diuiditur in nouem } \\
\text { modos. Primus modus est de } \\
\text { grammatica et de numero octo } \\
\text { partium orationis. Secundus est } \\
\text { de casu. Tertius de coniunctio- } \\
\text { ne. Quartus de declinatione. } \\
\text { Quintus de genere. Sextus de } \\
\text { regimine. Septimus de construc- } \\
\text { tione. Octauus de orthographia. } \\
\text { Nonus est de figura. }\end{array}$ & Cento forme \\
\hline $\begin{array}{l}\text { 6. III.77 Ars Brevis } \\
\text { (Pisa 1/1308) } \\
\text { (ROL XII, } \\
\text { p. 236) }\end{array}$ & $\begin{array}{l}\text { 88. Grammatica est ars inue- } \\
\text { niendi modum recte loquendi } \\
\text { recteque scribendi. }\end{array}$ & Cento forme \\
\hline $\begin{array}{l}\text { 7. IV.19 Ars mystica } \\
\text { theologiae et } \\
\text { philosophiae } \\
\text { (Parigi 11/1309) } \\
\text { (ROL V, p. 465) }\end{array}$ & $\begin{array}{l}\text { Grammatica est scientia recte } \\
\text { loquendi. Cuius subiectum est } \\
\text { oratio, in se et in suis partibus } \\
\text { considerata. Constructio autem, } \\
\text { quae tractatur a grammatico, } \\
\text { potest inquiri per tertiam circu- } \\
\text { lationem et per decem regulas } \\
\text { generales. }\end{array}$ & IX, 2 De artis liberalibus \\
\hline
\end{tabular}

